\title{
Clinical Application Research on the Use of Carnation Photon Therapeutic Apparatus in Treatment for Acute Postpartum Mastitis
}

\author{
Miao MIAO, Chun-Ni FAN, Ning LIU, Guang SUN* \\ Breast Surgery Department of China-Japan Union Hospital of Jilin University, \\ No.169,Xiantai Street Changchun Jilin Province,130021. \\ ${ }^{*}$ Corresponding author
}

Keywords: Photon therapeutic apparatus, Mastitis, Efficiency.

\begin{abstract}
Purpose: To investigate the clinical effect of photon therapeutic apparatus in the treatment of acute postpartum mastitis patients. Method: 48 cases of acute postpartum mastitis patients were randomly divided into two groups, treatment group and control group. Patients in the treatment group were treated with Carnation photon therapeutic apparatus once a day, while combing with antibiotics. Patients in the control group were treated with antibiotics. The two groups of patients should stop breast-feeding during treatment, empty the milk and clean nipple. Two groups would be observed curative effect after 7 days of treatment and one month follow-up. Results: In treatment group, there are $83.33 \%$ patients show healed, $12.5 \%$ patients show progressed, and only $4.17 \%$ shows reappeared after one month. Conclusion: Experiment results show that in the treatment group, the total efficiency was obviously higher than that of the control group. Visibly, treating the mastitis with carnation photon therapeutic apparatus is significant effective. Carnation photon therapeutic apparatus can anti-inflammatory, anti-infective, promote skin lesions healing, analgesia, reduce the exudation, and maintain the role of wound dry, which is worth in clinical application.
\end{abstract}

\section{Introduction}

Mastitis is a common disease in postpartum women, which is an acute suppurative infection of female ducts and its surrounding connective tissues[1].Its clinical manifestation is that the local breast swollen, the skin temperature increased, accompanied with enlarged lymph nodes, fever and chills, and even affect the health of lactation women. Adequate diagnosis and treatment of the disease in women are necessary to avoid lactation failure, recurrent mastitis, breast abscess, and even death in some situations. The studies in humans find that about a third of all lactating women will suffer from mastitis, where the clinical form of the disease is a primary reason why mothers will stop breast feeding [2].Most clinicians used antibiotics to treat mastitis, but many women are concerned that antibiotic therapy may affect the quality of infant breast feeding. Therefore, it is urgent to find a new treatment method [3]. Carnation photon therapeutic apparatus is a relatively new type of physiotherapy device, which is a specific wavelength of high power red photon penetration of $3-5 \mathrm{~cm}$, the energy sent to human tissue cells, a series of integrated biological effects, including photochemical effects, photoelectric effect, light pressure effect, light stimulation effect, light and heat effect, etc, to promote the cell tissue cell metabolism and granulation growth, thereby shortening wound healing process and improve the quality of wound healing[4]. Carnation photon therapeutic apparatus have certain curative effect in the treatment of 
pain, and anti-inflammation. This instrument has now been introduced into our hospital to treat acute mastitis, and achieved good treatment effect.

\section{Clinical Data}

\section{General Information}

All the patients we chose were lactating female, aged 20-35 (26.83 \pm 6.12$)$ years old. Course of mastitis is among 1 to 7 (3.12 \pm 0.23$)$ days. Of these patients, 42 cases were unilateral and 6 cases were bilateral. The average diameter of lesions area was $4.12 \pm 1.12 \mathrm{~cm}^{2}$.According to the order of admission, 24 cases were randomly divided into treatment group and 24 cases of control group. Those groups of cases such as gender, age, course of the disease, trauma based indexes $(\mathrm{P}>0.05)$, showed no statistical difference. Correlation analysis form is as below.

Treatment. Patients in the treatment group were treated with Carnation photon therapeutic apparatus once a day, 20 minutes each time, while combing with antibiotics. Patients in the control group were treated with antibiotics, 2 times a day. The two groups of patients should stop breast-feeding during treatment, empty the milk and clean nipple. Two groups would be observed curative effect after 7 days of treatment and one month follow-up.

Efficacy Determination. Healed: The symptoms were completely recovered, the breast lump was completely disappeared, and the breast milk was discharged smoothly, with no recurrence.

Progressed: The patient's symptoms and breast lumps are not improved or even worse. Mastitis has persisted into breast abscess, antibiotic or photon therapeutic apparatus is invalid, need to be treated by surgical resection.

Reappeared: Symptoms disappeared after treatment, after one month follow-up, mastitis reappeared.

Statistical Processing. Application SPSS17.0 statistical software, measuring data to $\mathrm{X} \pm \mathrm{S}$ said, using $\mathrm{t}$ test analysis; Count data used chi-square test, $\mathrm{P}<0.05$ for the difference was statistically significant.

\section{Results}

\section{Comparison of Clinical Efficacy Between the Two Groups}

We compare the two groups of patients' clinical curative effect after 7 days of treatment and one month follow-up. The results show that: In treatment group, there are 24 patients, among them, 20 cases show healed $(83.33 \%), 3$ cases show progressed (12.50\%), only 1 case shows reappeared after one month(4.17\%). At the control group, 12 of 24 cases show healed (50\%), 6 cases show progressed $(25 \%), 6$ case shows reappeared after one month $(25 \%)$. The treatment group compared with control group, with statistical significance $(\mathrm{p}<0.05)$. The results show in Table 1.

Table 1. Comparison of clinical efficacy between the two groups

\begin{tabular}{|c|c|c|c|c|c|c|c|}
\hline Group & \multirow{2}{*}{$\begin{array}{c}\text { Case } \\
\text { number(n) }\end{array}$} & \multicolumn{2}{|c|}{ Healed } & \multicolumn{2}{c|}{ Improved } & \multicolumn{2}{c|}{ Reappeared } \\
\cline { 3 - 8 } & & $\mathrm{n}$ & Rate(\%) & $\mathrm{n}$ & Rate(\%) & $\mathrm{n}$ & Rate(\%) \\
\hline Treatment & 24 & 20 & 83.33 & 3 & 12.50 & 1 & 4.17 \\
\hline Control & 24 & 12 & 50.00 & 6 & 25.00 & 6 & 25.00 \\
\hline
\end{tabular}




\section{Discussion}

Mastitis is an acute suppurative infection, which occurs in the process of galactostasis, bacteria get into the mammary gland through the nipple road. Most clinician often advocates antibiotic treatment. Because of concerns about antibiotic in milk and affect the baby, some maternal would stop breastfeeding, which may lead to milk stasis, and thus aggravate the inflammation, and even induce breast abscess. Therefore to seek a positive treatment method which will not affect breastfeeding is particularly urgent. Carnation photon therapeutic apparatus(Shenzhen Pumen science and Technology Co., Ltd. production) with the $630 \mathrm{~nm}$ of high powered, which could anti inflammatory, anti infective, promote skin lesions healing, analgesia, reduce the exudation, and maintain the role of wound dry [5].This apparatus can significantly improve the phagocytic function of leukocyte, enhance the immunity of the organism. And at the same time, it can help to improve the aerobic respiration, promote cell metabolism, and accelerate wound healing and disease rehabilitation. Carnation photon therapeutic apparatus can reduce 5-HT of the disease site, and thus produce a good analgesic effect [6]. This experiment has contrasted and observed the Carnation photon therapeutic apparatus and antibiotics were used in the treatment of mastitis. Treatment results show that in the treatment group, the total efficiency was obviously higher than that of the control group. Visibly, treating the acute mastitis with Carnation photon therapeutic apparatus has significant effect. The results show in Figure 1.

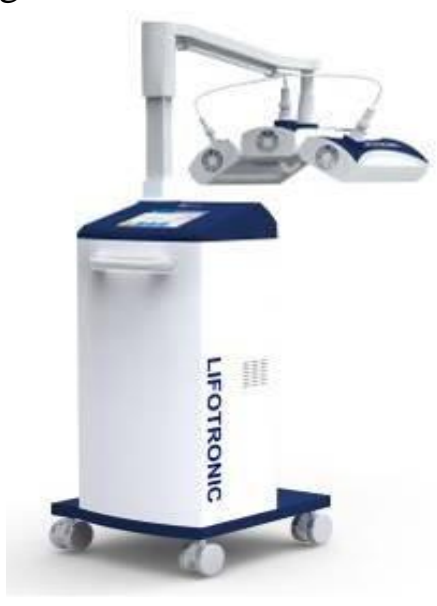

A

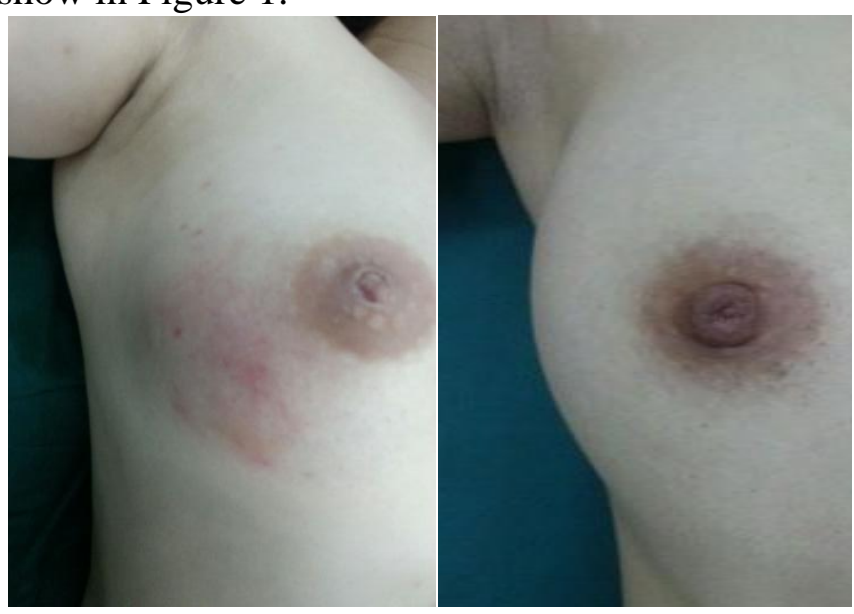

B
C

Figure 1. A. The acute postpartum mastitis patient. B. The photon therapeutic apparatus C.The breast shows healed.

\section{References}

[1] Aitken SL, Cod CM, Sordillo LM et a1. Immunopathology of mastitis insights into disease recognition and resolution[J].Journal of mammary gland biology and neoplasia,2011,16(4):291.

[2] Foxman B, D'Arcy H, Gillespie B,Bobo JK, Schwartz K.Lactation mastitis: occurrence and medical management among 946 breastfeeding women in the United States. Am J Epidemiol. 2002;155(2):103-14.

[3] A1 Khawari HA,AI Manfouhi HA, Madda JP et a1.Radiologic features of granulomatous mastitis.[J].The breast journal,2011,17(6):645.

[4] Stephens F,Bick DA.National pilot to implement pressure ulceguide lines,results of the baseline audit.Br J Community Nurs,2002,9:4. 
[5] M arcia Grant, Betty Ferrell, Grace Dean, et al. Revision and Psychometric Testing of the City of Hope Quality of Life-Ostomy Questionnaire [J].Quality of Life Reaserch , 2004,13(8):1445-1457.

[6] JuretaW1 Horton:Free redicals and lip id peroxidation mediated injury in burn trauma: the role of antioxidant therapy[J]1 Trauma,2003,189 (122):752881. 